\title{
A Soliton Hierarchy Associated with a Spectral Problem of 2nd Degree in a Spectral Parameter and Its Bi-Hamiltonian Structure
}

\author{
Yuqin Yao, ${ }^{1,2}$ Shoufeng Shen, ${ }^{2,3}$ and Wen-Xiu $\mathrm{Ma}^{2}$ \\ ${ }^{1}$ Department of Applied Mathematics, China Agricultural University, Beijing 100083, China \\ ${ }^{2}$ Department of Mathematics and Statistics, University of South Florida, Tampa, FL 33620, USA \\ ${ }^{3}$ Department of Applied Mathematics, Zhejiang University of Technology, Hangzhou 310023, China \\ Correspondence should be addressed to Yuqin Yao; yyqinw@126.com
}

Received 22 January 2016; Accepted 15 March 2016

Academic Editor: Boris G. Konopelchenko

Copyright (C) 2016 Yuqin Yao et al. This is an open access article distributed under the Creative Commons Attribution License, which permits unrestricted use, distribution, and reproduction in any medium, provided the original work is properly cited.

Associated with $\widetilde{\mathrm{so}}(3, R)$, a new matrix spectral problem of 2 nd degree in a spectral parameter is proposed and its corresponding soliton hierarchy is generated within the zero curvature formulation. Bi-Hamiltonian structures of the presented soliton hierarchy are furnished by using the trace identity, and thus, all presented equations possess infinitely commuting many symmetries and conservation laws, which implies their Liouville integrability.

\section{Introduction}

Consider an evolution equation

$$
u_{t}=K(u)=K\left(u, u_{x}, \ldots\right),
$$

where the field function $u(x, t)$ is in a linear space $S$ and $K(u)=K\left(u, u_{x}, \ldots\right)$ is a suitable $C^{\infty}$ vector field. Our starting point is to determine isospectral deformations of a linear spatial spectral problem

$$
\phi_{x}=U(u, \lambda) \phi \text {, }
$$

where $U$ is a square spectral matrix and $\lambda$ is a spectral parameter [1-3]. With the linear spectral problem (2), let us associate an auxiliary temporal spectral problem

$$
\phi_{t}=V(u, \lambda) \phi
$$

where $V$ is a square matrix of the same order as $U$. The compatibility condition of (2) and (3) is the zero curvature equation

$$
U_{t}-V_{x}+[U, V]=0
$$

If an evolution equation (1) can be presented by such a zero curvature equation, we call it a soliton equation, and $U$ and $V$ a Lax pair of (1). In general, for a well-chosen spectral matrix $U$, we can obtain, from different particular choices of $V$, a hierarchy of soliton equations:

$$
u_{t}=J \frac{\delta \mathscr{H}_{n}}{\delta u}
$$

where $J$ is a Hamiltonian operator and $\mathscr{H}_{n}$ 's are conserved functionals [1, 3, 4]. Many well-known examples of such soliton hierarchies are presented within the zero curvature formulation, which include the AKNS hierarchy $[2,5]$, the Kaup-Newell hierarchy $[6,7]$, the Wadati-Konno-Ichikawa hierarchy [8], the coupled Harry-Dym hierarchy [9], and the Dirac hierarchy [10].

Recently, the three-dimensional real special orthogonal Lie algebra $s o(3, \mathbb{R})$ has been used to construct soliton hierarchies [11-13]. The Lie algebra so $(3, \mathbb{R})$ consists of $3 \times 3$ trace-free, skew-symmetric matrices and has the basis

$$
e_{1}=\left(\begin{array}{ccc}
0 & 0 & -1 \\
0 & 0 & 0 \\
1 & 0 & 0
\end{array}\right),
$$




$$
\begin{aligned}
& e_{2}=\left(\begin{array}{ccc}
0 & 0 & 0 \\
0 & 0 & -1 \\
0 & 1 & 0
\end{array}\right), \\
& e_{3}=\left(\begin{array}{lll}
0 & -1 & 0 \\
1 & 0 & 0 \\
0 & 0 & 0
\end{array}\right),
\end{aligned}
$$

with the commutator relations:

$$
\begin{aligned}
& {\left[e_{1}, e_{2}\right]=e_{3},} \\
& {\left[e_{2}, e_{3}\right]=e_{1},} \\
& {\left[e_{3}, e_{1}\right]=e_{2} .}
\end{aligned}
$$

The derived algebra $[\operatorname{so}(3, \mathbb{R}), \operatorname{so}(3, \mathbb{R})]$ is $\operatorname{so}(3, \mathbb{R})$ itself. This is one of only two three-dimensional real Lie algebras with a three-dimensional derived algebra. The other one is $\operatorname{sl}(2, \mathbb{R})$, which has been widely used in studying soliton equations [2, $6,8,12-15]$. The corresponding matrix loop algebra is defined by

$$
\begin{aligned}
\widetilde{\mathrm{so}} & (3, \mathbb{R}) \\
& =\left\{\sum_{i \geq 0} M_{i} \lambda^{n-i} \mid M_{i} \in \operatorname{so}(3, \mathbb{R}), i \geq 0, n \in \mathbb{Z}\right\},
\end{aligned}
$$

that is, the space of all Laurent series in $\lambda$ with a finite number of nonzero terms of positive powers of $\lambda$ and coefficient matrices in so $(3, \mathbb{R})$. Particular examples of this matrix loop algebra $\widetilde{\mathrm{sO}}(3, \mathbb{R})$ contain the following linear combinations:

$$
\lambda^{m} e_{1}+\lambda^{n} e_{2}+\lambda^{l} e_{3}
$$

with arbitrary integers $m, n, l$.

Soliton hierarchies possess many nice properties, for instance, Lax representations or zero curvature representations, Hamiltonian structures, infinitely many conservation laws, and infinitely many symmetries. So it is interesting to search for new soliton hierarchies associated with a particular Lie algebra. In this paper, we would like to construct a spectral problem of 2 nd degree in a spectral parameter, based on the matrix loop algebra $\widetilde{\mathrm{so}}(3, \mathbb{R})$. A hierarchy of commuting bi-Hamiltonian soliton equations will be generated from associated zero curvature equations. Bi-Hamiltonian structures will be furnished by using the trace identity, and thus, the resulting hierarchy possesses infinitely commuting symmetries and conservation laws. A conclusion will be given in the last section.

\section{A Spectral Problem and Its Soliton Hierarchy}

To obtain a soliton hierarchy, we introduce a new spectral problem

$$
\phi_{x}=U(u, \lambda) \phi, \quad u=\left(\begin{array}{l}
p \\
q
\end{array}\right), \phi=\left(\begin{array}{l}
\phi_{1} \\
\phi_{2} \\
\phi_{3}
\end{array}\right),
$$

where the spectral matrix $U$ is defined by

$$
\begin{aligned}
U & =\lambda^{2} q e_{1}+\lambda e_{2}+\lambda p e_{3} \\
& =\left(\begin{array}{ccc}
0 & -\lambda p & -\lambda^{2} q \\
\lambda p & 0 & -\lambda \\
\lambda^{2} q & \lambda & 0
\end{array}\right) \in \widetilde{\text { SO }}(3, \mathbb{R}) .
\end{aligned}
$$

Once a matrix spectral problem is chosen, we apply the generating procedure $[1,3]$ to work out a soliton hierarchy associated with the spectral problem. First, we solve the stationary zero curvature equation

$$
W_{x}=[U, W] \text {. }
$$

If we assume $W$ to be

$$
W=a e_{1}+b e_{2}+c e_{3}=\left(\begin{array}{ccc}
0 & -c & -a \\
c & 0 & -b \\
a & b & 0
\end{array}\right),
$$

then (12) gives

$$
\begin{aligned}
& a_{x}=\lambda(c-p b) \\
& b_{x}=-\lambda^{2} q c+\lambda p a, \\
& c_{x}=-\lambda a+\lambda^{2} q b .
\end{aligned}
$$

Further, let $a, b$, and $c$ possess the Laurent expansions:

$$
\begin{aligned}
& a=\sum_{i \geq 0} a_{i} \lambda^{-2 i}, \\
& b=\sum_{i \geq 0} b_{i} \lambda^{-2 i-1}, \\
& c=\sum_{i \geq 0} c_{i} \lambda^{-2 i-1},
\end{aligned}
$$

and then, the system (14) leads to

$$
\begin{aligned}
& a_{i x}=c_{i}-p b_{i}, \\
& b_{i x}=p a_{i+1}-q c_{i+1}, \\
& c_{i x}=q b_{i+1}-a_{i+1}, \\
& \quad i \geq 0 .
\end{aligned}
$$


Using the above recursion relations (16) and taking the initial data

$$
\begin{aligned}
& a_{0}=1, \\
& b_{0}=\frac{1}{q}, \\
& c_{0}=\frac{p}{q},
\end{aligned}
$$

the sequences of $\left\{a_{i}, b_{i}, c_{i} \mid i \geq 1\right\}$ are determined uniquely provided that we take zero constants of integration. The first two sets are

$$
\begin{aligned}
a_{1} & =-\frac{1+p^{2}}{2 q^{2}} \\
b_{1} & =\frac{2 q p_{x}-2 p q_{x}-p^{2}-1}{2 q^{3}} \\
c_{1}= & \frac{2 q_{x}-p-p^{3}}{2 q^{3}} ; \\
a_{2} & =\frac{3+6 p^{2}+3 p^{4}-8 q p_{x}}{8 q^{4}}, \\
b_{2} & =\frac{1}{8 q^{5}}\left[3\left(1+p^{2}\right)\left(1+p^{2}-4 q p_{x}+4 p q_{x}\right)-24 q_{x}^{2}\right. \\
& \left.+8 q q_{x x}\right] \\
c_{2} & =\frac{1}{8 q^{5}}\left[6 p^{3}+3 p^{5}-12 p^{2} q_{x}+12\left(2 q p_{x}-1\right) q_{x}\right. \\
& \left.-8 q^{2} p_{x x}+p\left(3-24 q_{x}^{2}+8 q q_{x x}\right)\right] .
\end{aligned}
$$

From (16), we have

$$
\begin{aligned}
& \lambda\left(\lambda^{2 m+1} W\right)_{+x}-\left[U, \lambda\left(\lambda^{2 m+1} W\right)_{+}\right] \\
& \quad=\lambda\left(p a_{m+1}-q c_{m+1}\right) e_{2}+\lambda\left(q b_{m+1}-a_{m+1}\right) e_{3},
\end{aligned}
$$

$m \geq 0$,

where $p_{+}$denotes the polynomial part of $p$. However, a direct calculation gives

$$
U_{t}=\lambda p_{t} e_{3}+\lambda^{2} q_{t} e_{1}
$$

So in order to work out a soliton hierarchy, we should introduce modification terms $\Delta_{m}, m \geq 0$. Suppose

$$
\Delta_{m}=\lambda^{2} \delta_{1 m} e_{1}+\lambda \delta_{2 m} e_{2}+\lambda \delta_{3 m} e_{3}, \quad m \geq 0 ;
$$

we have

$$
\begin{aligned}
\Delta_{m x}-\left[U, \Delta_{m}\right]= & \left(\lambda^{2} \delta_{3 m}-\lambda^{2} p \delta_{2 m}+\lambda^{2} \delta_{1 m x}\right) e_{1} \\
& +\left(\lambda^{3} p \delta_{1 m}-\lambda^{3} q \delta_{3 m}+\lambda \delta_{2 m x}\right) e_{2} \\
& +\left(\lambda^{3} q \delta_{2 m}-\lambda^{3} \delta_{1 m}+\lambda \delta_{3 m x}\right) e_{3} .
\end{aligned}
$$

Based on (19)-(22), $\delta_{1 m}, \delta_{2 m}$, and $\delta_{3 m}$ should satisfy

$$
\begin{aligned}
p \delta_{1 m}-q \delta_{3 m} & =0, \\
q \delta_{2 m}-\delta_{1 m} & =0, \\
\delta_{2 m x}+\left(p a_{m+1}-q c_{m+1}\right) & =0,
\end{aligned}
$$

$$
m \geq 0
$$

The third equation in (23) gives $\delta_{2 m x}=q c_{m+1}-p a_{m+1}=-b_{m x}$, so $\delta_{2 m}=-b_{m}, \delta_{1 m}=-q b_{m}$, and $\delta_{3 m}=-p b_{m}$; that is,

$$
\begin{aligned}
\Delta_{m} & =\lambda^{2} \delta_{1 m} e_{1}+\lambda \delta_{2 m} e_{2}+\lambda \delta_{3 m} e_{3} \\
& =\left(\begin{array}{ccc}
0 & \lambda p b_{m} & \lambda^{2} q b_{m} \\
-\lambda p b_{m} & 0 & \lambda b_{m} \\
-\lambda^{2} q b_{m} & -\lambda b_{m} & 0
\end{array}\right), \quad m \geq 0 .
\end{aligned}
$$

As usual, we define

$$
V^{[m]}=\lambda\left(\lambda^{2 m+1} W\right)_{+}+\Delta_{m}, \quad m \geq 0,
$$

and then the corresponding zero curvature equations

$$
U_{t_{m}}-V_{x}^{[m]}+\left[U, V^{[m]}\right]=0, \quad m \geq 0,
$$

give rise to a soliton hierarchy

$$
u_{t_{m}}=K_{m}=\left(\begin{array}{l}
p \\
q
\end{array}\right)_{t_{m}}=\left(\begin{array}{c}
c_{m x}-\left(p b_{m}\right)_{x} \\
-\left(q b_{m}\right)_{x}
\end{array}\right), \quad m \geq 0 .
$$

Remark 1. The Lax pair for the hierarchy (27) is given by (2) and (3), where $U$ and $V$ are determined by (11) and (25). This implies that the hierarchy (27) is integrable in the Lax sense.

The first nonlinear system in this soliton hierarchy (27) is as follows:

$$
u_{t_{1}}=\left(\begin{array}{l}
p \\
q
\end{array}\right)_{t_{1}}=\left(\begin{array}{c}
\frac{1}{q^{4}}\left[\left(1+p^{2}\right)\left(q q_{x x}-3 q_{x}^{2}\right)-q^{2}\left(p_{x}^{2}+p p_{x x}\right)+4 p q p_{x} q_{x}\right] \\
\frac{1}{q^{3}}\left[\left(2 q p_{x}-1\right) q_{x}+p q\left(p_{x}+q_{x x}\right)-p^{2} q_{x}-q^{2} p_{x x}-2 p q_{x}^{2}\right]
\end{array}\right)
$$




\section{Bi-Hamiltonian Structures and Liouville Integrability}

In this subsection, we will show that the soliton hierarchy (27) is Liouville integrable [16-18]. First, let us establish biHamiltonian structures for the hierarchy (27) by using the trace identity $[11,19]$ or the variational identity $[20,21]$ :

$$
\begin{array}{r}
\frac{\delta}{\delta u} \int \operatorname{tr}\left(\frac{\partial U}{\partial \lambda} W\right) d x=\lambda^{-\gamma} \frac{\partial}{\partial \lambda} \lambda^{\gamma} \operatorname{tr}\left(\frac{\partial U}{\partial u} W\right), \\
\gamma=-\frac{\lambda}{2} \frac{d}{d \lambda} \ln \left|\operatorname{tr}\left(W^{2}\right)\right| .
\end{array}
$$

It is easy to find that

$$
\begin{aligned}
& \frac{\partial U}{\partial \lambda}=\left(\begin{array}{ccc}
0 & -p & -2 \lambda q \\
p & 0 & -1 \\
\lambda q & 1 & 0
\end{array}\right), \\
& \frac{\partial U}{\partial p}=\left(\begin{array}{ccc}
0 & -\lambda & 0 \\
\lambda & 0 & 0 \\
0 & 0 & 0
\end{array}\right) \\
& \frac{\partial U}{\partial q}=\left(\begin{array}{ccc}
0 & 0 & -\lambda^{2} \\
0 & 0 & 0 \\
\lambda^{2} & 0 & 0
\end{array}\right) .
\end{aligned}
$$

Thus, we have

$$
\begin{aligned}
& \operatorname{tr}\left(W \frac{\partial U}{\partial \lambda}\right)=-4 \lambda q a-2 p c-2 b \\
& \operatorname{tr}\left(W \frac{\partial U}{\partial p}\right)=-2 \lambda c \\
& \operatorname{tr}\left(W \frac{\partial U}{\partial q}\right)=-2 \lambda^{2} a .
\end{aligned}
$$

Plugging these quantities into the trace identity (29) gives

$$
\frac{\delta}{\delta u} \int(-4 \lambda q a-2 p c-2 b) d x=\lambda^{-\gamma} \frac{\partial}{\partial \lambda} \lambda^{\gamma}\left(\begin{array}{c}
-2 \lambda c \\
-2 \lambda^{2} a
\end{array}\right)
$$

Balancing the coefficients of $\lambda^{-2 m-1}$ in the above equation leads to

$$
\begin{array}{r}
\frac{\delta}{\delta u} \int\left(2 q a_{m+1}+p c_{m}+b_{m}\right) d x=(\gamma-2 m)\left(\begin{array}{c}
c_{m} \\
a_{m+1}
\end{array}\right) \\
m \geq 0 .
\end{array}
$$

The identity with $m=1$ yields $\gamma=0$ and thus we have

$$
\frac{\delta}{\delta u} \mathscr{H}_{m}=\left(\begin{array}{c}
c_{m} \\
a_{m+1}
\end{array}\right), \quad m \geq 0
$$

with the Hamiltonian functions being defined by

$$
\begin{aligned}
\mathscr{H}_{0} & =\int \frac{1+p^{2}}{2 q} d x, \\
\mathscr{H}_{m} & =-\int \frac{2 q a_{m+1}+p c_{m}+b_{m}}{2 m} d x, \quad m \geq 1 .
\end{aligned}
$$

From (16), we have

$$
\begin{aligned}
b_{m} & =-\partial^{-1} q c_{m+1}+\partial^{-1} p a_{m+1} \\
& =-\partial^{-1} q\left(\partial a_{m+1}+p b_{m+1}\right)+\partial^{-1} p a_{m+1} \\
& =-\partial^{-1} q \partial a_{m+1}-\partial^{-1} p\left(c_{m x}+a_{m+1}\right)+\partial^{-1} p a_{m+1}, \\
& m \geq 0 .
\end{aligned}
$$

Thus the hierarchy (27) can be written as

$$
\begin{aligned}
u_{t_{m}} & =K_{m}=\left(\begin{array}{c}
c_{m x}-\left(p b_{m}\right)_{x} \\
-\left(q b_{m}\right)_{x}
\end{array}\right) \\
& =\left(\begin{array}{c}
c_{m x}+\partial p \partial^{-1} q \partial a_{m+1}+\partial p \partial^{-1} p \partial c_{m} \\
\partial q \partial^{-1} q \partial a_{m+1}+\partial q \partial^{-1} p \partial c_{m}
\end{array}\right) \\
& =J\left(\begin{array}{c}
c_{m} \\
a_{m+1}
\end{array}\right), \quad m \geq 0,
\end{aligned}
$$

where

$$
J=\left(\begin{array}{cc}
\partial+\partial p \partial^{-1} p \partial & \partial p \partial^{-1} q \partial \\
\partial q \partial^{-1} p \partial & \partial q \partial^{-1} q \partial
\end{array}\right)
$$

It can be verified that $J$ is skew symmetry and satisfies the Jacobi identity, so it is a Hamiltonian operator. It now follows that the soliton hierarchy (27) has the Hamiltonian structures

$$
u_{t_{m}}=K_{m}=J \frac{\delta \mathscr{H}_{m}}{\delta u}, \quad m \geq 0 .
$$

From the recursion relations (16), we can have

$$
\left(\begin{array}{c}
c_{m} \\
a_{m+1}
\end{array}\right)=\Psi\left(\begin{array}{c}
c_{m-1} \\
a_{m}
\end{array}\right), \quad m \geq 0
$$


where

$$
\Psi=\left(\begin{array}{cc}
\frac{p}{q} \partial & \partial+\frac{p}{q} \\
-\partial^{-1} \frac{1}{q} \partial \frac{1}{q} \partial-\partial^{-1} \frac{p}{q} \partial \frac{p}{q} \partial & -\partial^{-1} \frac{1}{q} \partial \frac{1}{q}-\partial^{-1} \frac{p}{q} \partial^{2}-\partial^{-1} \frac{p}{q} \partial \frac{p}{q}
\end{array}\right)
$$

It is easy to verify that $J \Psi=\Psi^{*} J$. Actually, we can show that the hierarchy (27) is bi-Hamiltonian:

$$
u_{t_{m}}=K_{m}=J \frac{\delta \mathscr{H}_{m}}{\delta u}=M \frac{\delta \mathscr{H}_{m-1}}{\delta u}, \quad m \geq 1,
$$

where the second Hamiltonian operator is

$$
M=J \Psi=\left(\begin{array}{cc}
0 & \partial^{2} \\
-\partial^{2} & -\partial
\end{array}\right) .
$$

Here $J$ and $M$ constitute a Hamiltonian pair. Particularly,

$$
\begin{aligned}
\left\{\mathscr{H}_{k}, \mathscr{H}_{l}\right\}_{J} & =\int\left(\frac{\delta \mathscr{H}_{k}}{\delta u}\right)^{T} J \frac{\delta \mathscr{H}_{l}}{\delta u} d x=0, \quad k, l \geq 0, \\
\left\{\mathscr{H}_{k}, \mathscr{H}_{l}\right\}_{M} & =\int\left(\frac{\delta \mathscr{H}_{k}}{\delta u}\right)^{T} M \frac{\delta \mathscr{H}_{l}}{\delta u} d x=0, \quad k, l \geq 0, \\
{\left[K_{k}, K_{l}\right] } & =K_{k}^{\prime}(u)\left[K_{l}\right]-K_{l}^{\prime}(u)\left[K_{k}\right]=0, \\
\quad & k, l \geq 0 .
\end{aligned}
$$

These commuting relations are also consequences of the Virasoro algebra of Lax operators [22, 23]. To sum up the above discussion, we obtain the following proposition and theorem.

Proposition 2. The soliton hierarchy (27) has infinitely many common commuting symmetries $\left\{J\left(\delta H_{m} / \delta u\right)\right\}_{m=1}^{\infty}$ and infinitely many conserved functions $\left\{H_{m}\right\}_{m=0}^{\infty}$.

Theorem 3. The soliton equations in the soliton hierarchy (27) are all integrable in Liouville sense.

\section{Concluding Remarks}

Based on the special orthogonal Lie algebra $\widetilde{\mathrm{so}}(3, \mathbb{R})$, the AKNS spectral matrix, the KN spectral matrix, and the WKI spectral matrix were presented in $[11,16,24]$, respectively. Those spectral matrices are

$$
\begin{aligned}
& U(u, \lambda)=\lambda e_{1}+q e_{2}+p e_{3}, \\
& U(u, \lambda)=\lambda^{2} e_{1}+\lambda q e_{2}+\lambda p e_{3}, \\
& U(u, \lambda)=\lambda e_{1}+\lambda q e_{2}+\lambda p e_{3}, \\
& U(u, \lambda)=(\lambda+q) e_{1}+p e_{2}+e_{3},
\end{aligned}
$$

where $u=(p, q)^{T}$ includes two dependent variables.
In our paper, the new spectral problem defined by (10) with (11), associated with $\widetilde{\mathrm{so}}(3, \mathbb{R})$, has been proposed and its corresponding soliton hierarchy has been worked out. The new soliton hierarchy is bi-Hamiltonian, and so, the resulting equations possess infinitely many commuting symmetries and conserved functionals, which implies that they are Liouville integrable. Furthermore, we will further research the integrable couplings and Darboux transformation of the integrable hierarchy (27).

It is particularly interesting to explore other types of spectral problems of even higher degree in a spectral parameter, associated with so $(3, \mathbb{R})$, which generate soliton hierarchies. All those studies should provide insightful thoughts to classify multicomponent integrable systems.

\section{Competing Interests}

The authors declare that they have no competing interests.

\section{Acknowledgments}

This work is supported in part by NSF under the Grant DMS-1301675, National Natural Science Foundation of China (Grant nos. 11171175, 11201477, 11301179, 11271008, and 61072147), Zhejiang Innovation Project of China (Grant no. T200905), the First-Class Discipline of Universities in Shanghai and Shanghai University Leading Academic Discipline Project (no. A.13-0101-12-004), and China Scholarship Council.

\section{References}

[1] G. Z. Tu, "On a new hierarchy of Hamiltonian soliton equations," Journal of Mathematical Physics, vol. 24, no. 8, pp. 23052341, 1983.

[2] M. J. Ablowitz, D. J. Kaup, A. C. Newell, and H. Segur, "The inverse scattering transform-fourier analysis for nonlinear problems," Studies in Applied Mathematics, vol. 53, no. 8, pp. 249-315, 1974.

[3] W. X. Ma, "A new hierarchy of Liouville integrable generalized Hamiltonian equations and its reduction," Chinese Annals of Mathematics A, vol. 13, pp. 115-123, 1992.

[4] M. Antonowicz and S. Rauch-Wojciechowski, "How to construct finite-dimensional bi-Hamiltonian systems from soliton equations: Jacobi integrable potentials," Journal of Mathematical Physics, vol. 33, no. 6, pp. 2115-2125, 1992.

[5] Q. L. Zha, "A generalized AKNS hierarchy, bi-Hamiltonian structure, and Darboux transformation," Communications in Nonlinear Science and Numerical Simulation, vol. 17, no. 6, pp. 2319-2332, 2012. 
[6] D. J. Kaup and A. C. Newell, "An exact solution for a derivative nonlinear Schrödinger equation," Journal of Mathematical Physics, vol. 19, no. 4, pp. 798-801, 1978.

[7] Z. H. Yang and Y. B. Zeng, "On generating equations for the Kaup-Newell hierarchy," Applied Mathematics, vol. 22, no. 4, pp. 413-420, 2007.

[8] M. Wadati, K. Konno, and Y. H. Ichikawa, "New integrable nonlinear evolution equations," Journal of the Physical Society of Japan, vol. 47, no. 5, pp. 1698-1700, 1979.

[9] M. Antonowicz and A. P. Fordy, "Coupled Harry Dym equations with multi-Hamiltonian structures," Journal of Physics A: Mathematical and General, vol. 21, no. 5, pp. L269-L275, 1988.

[10] J. Zhang, F. You, and Y. Zhao, "A new super extension of Dirac hierarchy," Abstract and Applied Analysis, vol. 2014, Article ID 472101, 6 pages, 2014.

[11] W. X. Ma, "A spectral problem based on $\operatorname{so}(3, R)$ and its associated commuting soliton equations," Journal of Thematical Physics, vol. 54, no. 10, Article ID 103509, 2013.

[12] E. Fan, "A Liouville integrable Hamiltonian system associated with a generalized Kaup-Newell spectral problem," Physica A. Statistical Mechanics and its Applications, vol. 301, no. 1-4, pp. 105-113, 2001.

[13] Y. F. Zhang, "A generalized Boite-Pempinelli-Tu (BPT) hierarchy and its bi-Hamiltonian structure," Physics Letters A, vol. 317, no. 3-4, pp. 280-286, 2003.

[14] P. D. Lax, "Integrals of nonlinear equations of evolution and solitary waves," Communications on Pure and Applied Mathematics, vol. 21, pp. 467-490, 1968.

[15] M. Boiti, F. Pempinelli, and G. Z. Tu, "Canonical structure of soliton equations via isospectral eigenvalue problems," Nuovo Cimento B, vol. 79, no. 2, pp. 231-265, 1984.

[16] W. X. Ma, "An integrable counterpart of the D-AKNS soliton hierarchy from so(3, R)," Physics Letters A, vol. 378, no. 24, pp. 1717-1720, 2014.

[17] Y.-P. Sun and D.-Y. Chen, "A Liouville integrable hierarchy, symmetry constraint, new finite-dimensional integrable systems, involutive solution and expanding integrable models," Chaos, Solitons \& Fractals, vol. 29, no. 4, pp. 978-987, 2006.

[18] M. Baxter, S. R. Choudhury, and R. A. Van Gorder, "Zero curvature representation, bi-Hamiltonian structure, and an integrable hierarchy for the Zakharov-Ito system," Journal of Mathematical Physics, vol. 56, no. 6, Article ID 063503, 2015.

[19] G. Z. Tu, "The trace identity, a powerful tool for constructing the Hamiltonian structure of integrable systems," Journal of Mathematical Physics, vol. 30, no. 2, pp. 330-338, 1989.

[20] W.-X. Ma and M. Chen, "Hamiltonian and quasi-Hamiltonian structures associated with semi-direct sums of Lie algebras," Journal of Physics A: Mathematical and General, vol. 39, no. 34, pp. 10787-10801, 2006.

[21] F. K. Guo and Y. F. Zhang, "The quadratic-form identity for constructing the Hamiltonian structure of integrable systems," Journal of Physics A: Mathematical and General, vol. 38, no. 40, pp. 8537-8548, 2005.

[22] W. X. Ma, "The algebraic structures of isospectral Lax operators and applications to integrable equations," Journal of Physics A. Mathematical and General, vol. 25, no. 20, pp. 5329-5343, 1992.

[23] A. M. Semikhatov, "The Virasoro algebra in integrable hierarchies and the method of matrix models," Zhurnal Eksperimental'noi i Teoreticheskoi Fizik, vol. 101, pp. 779-827, 1992.

[24] S. F. Shen, L. Y. Jiang, Y. Y. Jin, and W. X. Ma, "New soliton hierarchies associated with the Lie algebra SO $(3, \mathbb{R})$ and their bi-Hamiltonian structure," Reports on Mathematical Physics, vol. 75, no. 1, pp. 113-133, 2015. 


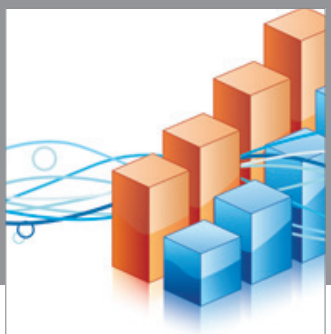

Advances in

Operations Research

vatem alat4

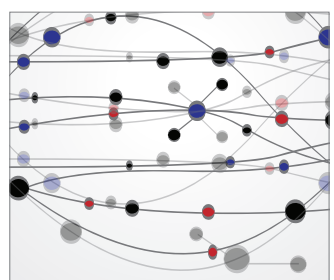

\section{The Scientific} World Journal
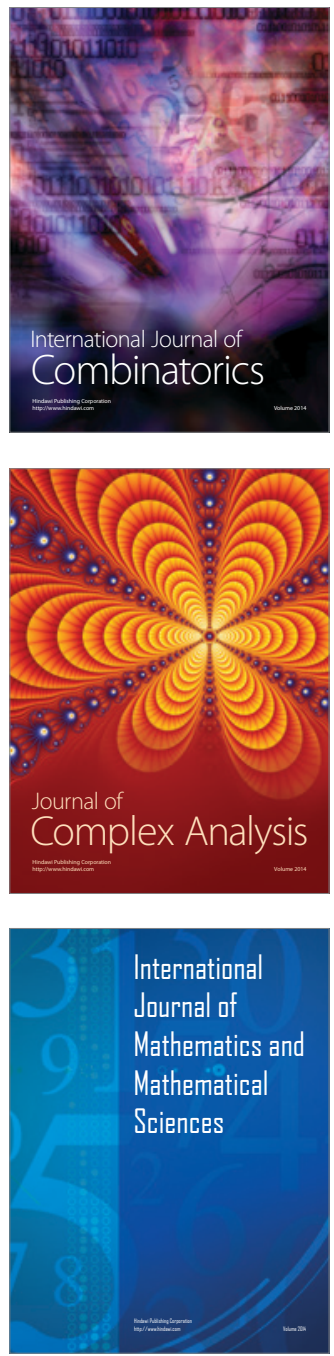
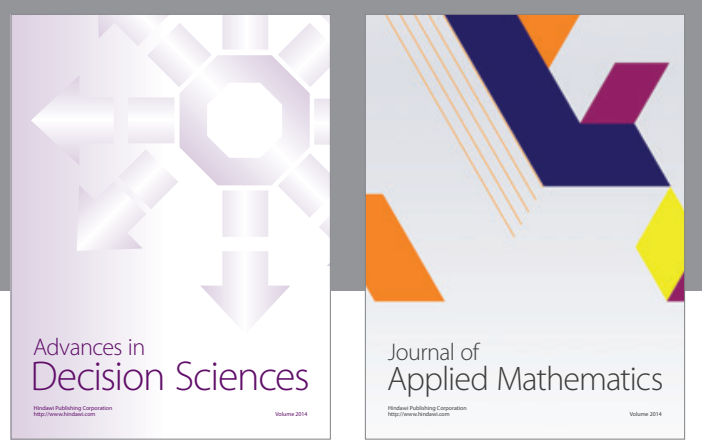

Algebra

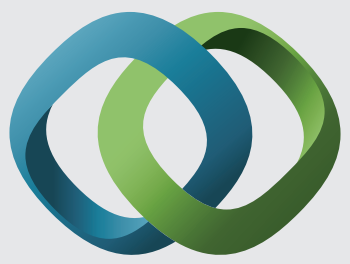

\section{Hindawi}

Submit your manuscripts at

http://www.hindawi.com
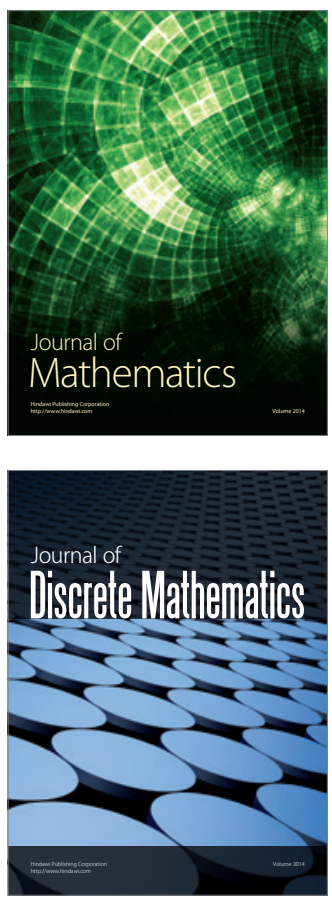

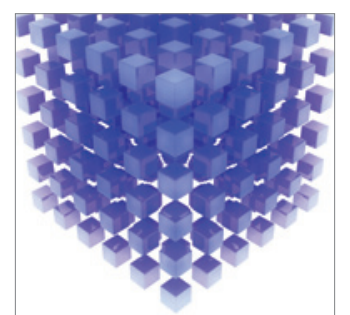

Mathematical Problems in Engineering
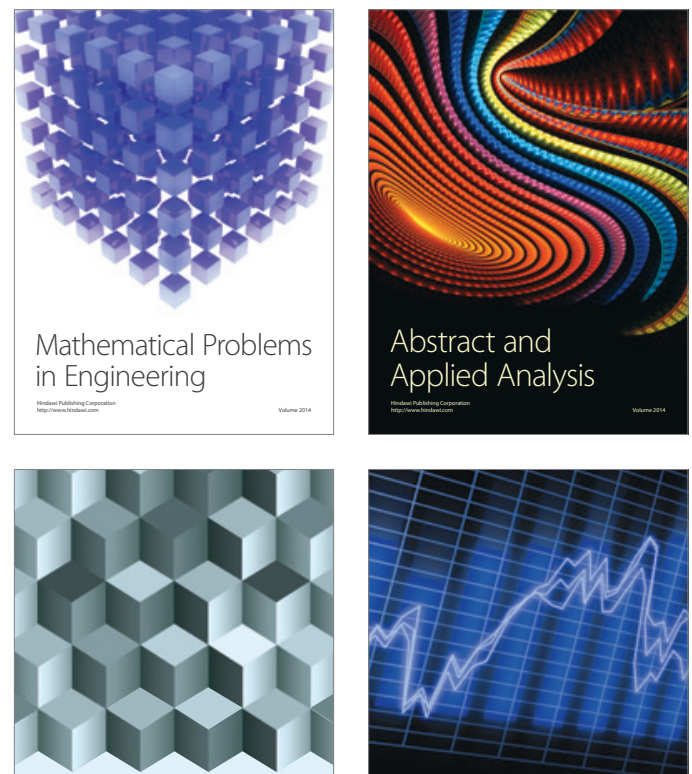

Journal of

Function Spaces

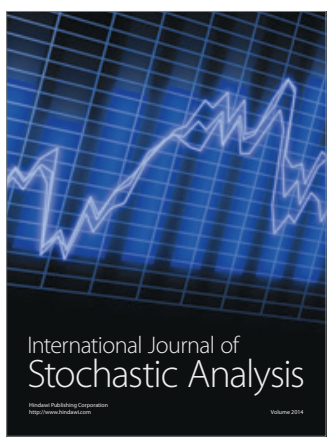

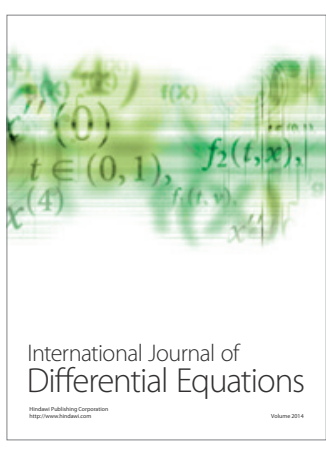
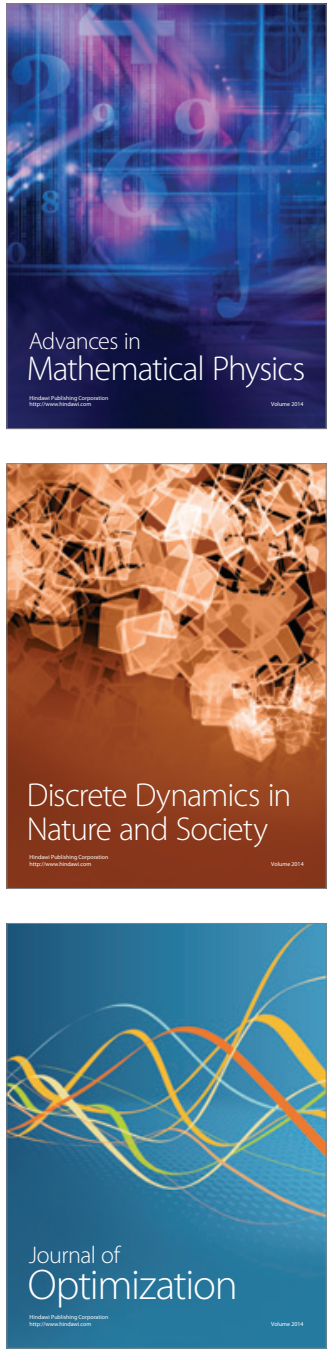